\title{
REPRESENTACIONES SOCIALES DEL ESTADO Y LA COMUNIDAD SOBRE LA REHABILITACIÓN DE JÓVENES CON ADICCIONES EN ZONAS URBANO MARGINALES DE GUAYAQUIL 2016-2019
}

\author{
Carlos Terán-Puente ${ }^{1}$, Milton Gross Albornoz $^{2}$ y María Aguayo Canchingre ${ }^{1}$ \\ 1Universidad Estatal de Milagro, UNEMI, Ecuador. cteranp@unemi.edu.ec; maguayoc@unemi.edu.ec \\ ${ }^{2}$ Pontificia Universidad Católica del Ecuador, PUCE, Ecuador. mgross@puce.edu.ec
}

\begin{abstract}
Resumen. El artículo explora las representaciones sociales de la comunidad y del Estado frente a la rehabilitación de jóvenes y adolescentes afectados por el consumo problemático de sustancias sujetas a fiscalización en las barriadas de Guayaquil. Entre 2016 y 2019, sucedieron una serie de incendios de pequeñas clínicas clandestinas para personas con adicciones. Hubo fallecidos y heridos entre quienes estaban internados para procesos de rehabilitación. El objetivo es analizar las representaciones sociales en la gestión de las adicciones como problema de salud pública. Metodológicamente, es una investigación descriptiva, longitudinal y cualitativa, hermenéutica. Resultados: Se evidencia un círculo reiterado de intervenciones desde la institucionalidad estatal frente al problema. Se evidencian posturas paralelas, discrepantes y hasta contradictorias frente a la rehabilitación de personas con adicciones. Una gestión estatal fundada en un paradigma fragmentario, punitivo, implementada con acento tecnocrático y administrativo, resulta desconectada de la problemática y visión de la comunidad, elude la participación comunitaria y no augura una solución. Se requiere revisar las representaciones sociales del Estado y su gestión de las adicciones, comprendiendo la urgencia de incluir la participación social como base del sistema sanitario y del mismo funcionamiento democrático.
\end{abstract}

Palabras-clave: Salud Pública; Gestión Sanitaria; Adicciones; Representaciones Sociales; Marginalidad Urbana; Participación Social.

\section{SOCIAL REPRESENTATIONS OF THE STATE AND COMMUNITY ON REHABILITATION OF YOUNG PEOPLE WHIT ADDICTIONS IN URBAN ZONES OF GUAYAQUIL 2016-2019}

\begin{abstract}
The article explores the social representations of the State and community regarding rehabilitation of youth and adolescents affected by the problematic consumption of substances subject to control in neighbourhoods of Guayaquil. Between 2016 and 2019, there were a series of fires in small clandestine clinics for people with addictions. There were deaths and injuries among those who were interned for rehabilitation processes. The article objective is to analyse social representations on addictions management as a public health problem. Methodologically, it is a descriptive, longitudinal and qualitative, hermeneutical investigation. Results: there is a repeated circle of interventions from the state institutions in face of the problem. Parallel, discrepant and even contradictory positions are evident about rehabilitation of people with addictions. A state management based on a fragmentary, punitive paradigm, implemented with a technocratic and administrative accent, is disconnected from the problems and vision of the community, avoids community participation and does not audit a solution. Conclusions: It is necessary to review the social representations of the State and its addiction management, understanding the urgency of including social participation as the basis of the health system and the same democratic functioning.
\end{abstract}

Keywords: Public Health; Health Management; Addictions; Social Representations; Urban Marginality; Social Participation. 


\section{INTRODUCCIÓN}

Desde la vertiente latinoamericana de la epidemiología (Filho \& Paim, 1999) conocida por un enfoque integrado y crítico de la salud pública, Edmundo Granda ${ }^{1}$, con amplia y profunda motivación, propone que salud colectiva es desarrollar las mejores ideas y acciones para apoyar la forja de una salud pública que pueda interpretar y mediar con conocimiento y eficacia en el mejoramiento y cuidado de los niveles de salud de la población (Granda, 2004). El presente artículo examina -con esta mirada- la gestión pública en la rehabilitación de personas con consumo problemático de sustancias sujetas a fiscalización, lo cual no puede ser valorado desde la pura estadística. Una serie de incendios, generalmente provocados en clínicas de desintoxicación ubicadas en zonas urbano-marginales, con fallecimiento y daño de las personas internadas, son hechos graves, más aún si son reiterados. Constituyen un atentado contra la dignidad y la vida de las personas, cuestionan el significado de la rectoría estatal de la salud pública que debe ser el aseguramiento de la vigencia y protección de los derechos humanos (Terán-Puente, 2019).

Apenas comenzado el 2019, en una barriada marginal de Guayaquil, 18 personas fallecieron y 16 quedaron heridas en el incendio intencional de una "clínica particular de rehabilitación para personas con adicciones". Se trata de servicios privados que ofrecen desintoxicar a personas con adicciones. A partir de este hecho, se investigaron casos similares referidos en numerosos reportajes impresos y audiovisuales publicados en medios de comunicación. Así, se encontró que entre 2016 y 2019, se produjeron 13 incendios en centros de rehabilitación, la mayoría intencionales, con un total de 29 personas fallecidas, incineradas o asfixiadas, 26 heridos reportados y un número indeterminado de personas, generalmente adolescentes y jóvenes, escapados de dichas clínicas en medio de los flagelos. Tan solo en 2019, hubo media docena de incendios, los más graves sucedieron en enero y octubre, con 28 personas fallecidas en total2.

La casi totalidad de los incendios son intencionales provocados por jóvenes internados con la finalidad de llamar la atención sobre sus condiciones de "tratamiento". Se conoce que los síndromes de abstinencia son "atendidos" con encierro en celdas, denominadas "lagarteras", en condiciones de hacinamiento. Muchas de estas personas son internadas sin su

\footnotetext{
${ }^{1}$ Médico-Cirujano, Master en Medicina Social. Planificador en Salud. 1946-2008. Autor de innumerables publicaciones y uno de los forjadores del pensamiento latinoamericano en la salud pública. Su obra ha merecido el reconocimiento de la academia nacional y de la Organización Panamericana de la Salud.

${ }^{2} \mathrm{El}$ incendio de enero 2019 (18 muertes y 16 heridos) tuvo amplio despliegue periodístico y repercusión pública. El incendio de octubre 2019 (10 muertes) pasó casi desapercibido por el contexto de una masiva protesta social violenta contra la política económica del gobierno. En ese mes, los medios reportan tres incendios más.
} 
consentimiento. Cada incendio es un llamado para que se los libere de este tipo de procedimientos. Es necesario resaltar que además de incendios con víctimas mortales y heridos, se reportan públicamente casos de suicidios de internos, otros fallecimientos presuntamente por sobredosis y episodios de agresiones entre las personas internadas en las clínicas de desintoxicación³. La serie de casos se ubica en un mismo entorno geo poblacional: son familias y comunidades de estratos empobrecidos que habitan asentamientos relativamente recientes, ciudadelas informales con jefes o jefas de hogar subempleadas o desempleadas, migrantes de otras provincias o de la zona rural, población empobrecida.

Según el Ministerio de Salud Pública, existen 40 centros especializados en el país, algunos con licencia condicionada a mejoras. Hay una oferta total de 1.319 camas para con internación (MSP, 2019). Se afirma que hay 743 centros ambulatorios, 28 unidades de salud mental hospitalaria y 12 centros residenciales. Según la entidad nacional responsable, 89\% de personas con adicciones son atendidas en servicios privados, mientras los centros públicos atienden 11\% de los casos (Secretaría Técnica de Drogas, 2017).

La serie de incidentes 2016 - 2019, incluye 13 incendios en 40 meses, lo que configura un escenario reiterado, un patrón social, ante lo cual hay una respuesta estatal que declarara públicamente el estatuto legal de los centros quemados, sobre todo si resultaren "clandestinos", o no autorizados; los clausura aún en medio de las cenizas, anuncia que se abrirán expedientes para sancionar posibles irregularidades dentro de la institución. Su respuesta vuelve y repite que la ciudadanía debe acudir solo a lugares autorizados por la ley. El incendio de enero $2019^{4}$, fue motivo de pública condolencia del presidente del país y hubo similares expresiones de las máximas autoridades. Ante este mismo caso, la titular del MSP compareció ante los medios y asumió su presencia normativa. Ofertó la construcción y funcionamiento de un megacentro de referencia nacional para tratamiento de adicciones 5 . Por otro lado, la agencia de control del MSP reforzó sus operativos regulares, recorrió los

${ }^{3}$ El Expreso. Edición impresa, 29 diciembre 2019.

${ }^{4} \mathrm{La}$ tragedia se registró en la cooperativa Proletarios sin tierra, en el Guasmo Sur de Guayaquil. Funcionaba en una angosta casa en el suburbio. Los residentes no lograron salir debido a que las puertas estaban cerradas. Luego de esa tragedia se reeditó el debate la falta de centros autorizados y la deficiencia de centros públicos. Fuente: Diario El Comercio, en:https://www.elcomercio.com/actualidad/fallecidos-incendio-adicciones-guayaquil.html.

5 Junto al Gobernador, la Ministra de Salud "anunció la construcción de un Gran Complejo de Atención Integral a personas con uso y consumo problemático de alcohol y otras drogas que será de referencia nacional y se ubicará en la ciudad de Guayaquil...". Fuente: https://www.salud.gob.ec/ministra-de-salud-anuncia-construccion-gran-complejode-atencion-en-salud-mental-y-nuevas-rutas-de-atencion-para-tratar-el-consumo-de-drogas/. 
barrios, ejecutó intervenciones de control de establecimientos, verificó los permisos de funcionamiento y clausuró establecimientos no autorizados ${ }^{6}$.

El municipio de Guayaquil, reconociendo el incremento de las adicciones en la ciudad, implementó su propio plan en coincidencia y complemento del programa del MSP. Tiene la misma lógica pero con una diferencia, es una "clínica ambulante" que irá de barrio en barrio ofertando consultas diarias, permaneciendo varios días en un mismo barrio. Este programa, junto con el MSP, asegura que logrará la desintoxicación, rehabilitación y reinserción social de jóvenes y adolescentes con consumo problemático de sustancias ${ }^{7}$. Los médicos generales y psicólogos de la clínica móvil tratarán hasta cien pacientes diarios. Los medios informaron: "...luego de que se suscribiera un convenio con el Ministerio de Salud para dar tratamiento a los consumidores... se formó una larga fila de jóvenes y sus familiares para obtener la atención gratuita" ${ }^{\text {. }}$

En la serie de incidentes relacionados, se constata un círculo reiterativo con varios nudos críticos:

i) Crecimiento exponencial de personas con adicciones (jóvenes, adolescentes);

ii) Familias que acuden a establecimientos no autorizados (89\%) y autorizados (11\%)

iii) Incendios reiterados intencionales en clínicas "clandestinas" (suicidios, fallecimientos);

iv) Respuesta institucional reiterada e invariable: llamado a cumplir la norma y reprobar lo ilegal. Se suma la oferta de ampliar servicios públicos.

\subsection{Entorno legal}

La Constitución de Ecuador, 2008, trata el consumo de sustancias sujetas a fiscalización con un criterio orientado a la descriminalización de los usuarios, la proporcionalidad de las penas para delitos de drogas y una reorientación del enfoque penal hacia uno de salud pública. El artículo 364, establece que las adicciones son un problema de salud pública y que le corresponde al estado desarrollar programas coordinados de información, prevención

6La Agencia de Aseguramiento de la Calidad de los Servicios de Salud y Medicina Prepagada (ACESS), en 2019, que realizó cientos de clausuras y, específicamente en la zona 8 (correspondiente a Guayaquil), realizó 31 operativos y 29 clausuras de centros de rehabilitación. Fuente: Diario EL COMERCIO en: https://www.elcomercio.com

7La Alcaldía de Guayaquil inauguró la primera clínica móvil de desintoxicación de drogas de la ciudad y del país, en la que se brindará atención médica y psicológica gratuita a personas que están sumergidas en el problema de las drogas. "A través de un convenio con el Ministerio de Salud Pública lograremos salvar el futuro de la juventud. Desde la Municipalidad trabajaremos en desintoxicación, el MSP en rehabilitación y juntos trabajaremos en su reinserción social", dijo la alcaldesa de Guayaquil". Fuente: Redacción Médica. Edición nacional, 13 de agosto 2019.

${ }_{8}$ El Universo, edición del 12 de agosto 2019 
y control del consumo de alcohol, tabaco y sustancias estupefacientes y psicotrópicas; así como ofrecer tratamiento y rehabilitación a los consumidores ocasionales, habituales y problemáticos. En ningún caso se permitirá su criminalización ni se vulneran sus derechos constitucionales" (Ecuador, 2008).

El Código Orgánico Integral Penal, establece parámetros para clasifica las actividades consideradas ilícitas y establece penas proporcionales en función de los delitos, diferencia entre grandes y pequeños traficantes; gradúa las penas en función del rol en los actos ilícitas; y distingue personas usuarias de los personas traficantes. El cultivo comercial de plantas con propiedades psicoactivas queda prohibido y, en consecuencia, descriminaliza el cultivo para consumo personal (Asamblea Nacional del Ecuador, 2004). El Plan Nacional de Prevención Integral y Control del Fenómeno Socioeconómico de las Drogas (2017-2021), de la Secretaría Técnica de Prevención Integral de Drogas, se orienta a prevenir el uso y consumo de drogas, a brindar atención integral e intersectorial a las personas que presentan uso o consumo problemático, a disminuir la oferta de drogas destinadas al consumo interno y a prevenir la producción ilícita de drogas. Para la atención integral e intersectorial a las personas en consumo problemático de drogas propone un abordaje programático que incluya adecuado tratamiento con la finalidad de rehabilitarlo de su padecimiento y garantizar, de esta manera, su reinserción social (Secretaría Técnica de Drogas, 2017)

Teóricamente, las características de este abordaje observan principios de no criminalización, corresponsabilidad, intersectorialidad, participación ciudadana, inclusión, interculturalidad, género, prevención integral para toda una vida, y enfoque intergeneracional, fundamentados en la mejor evidencia científica, y que permitan medir su impacto social. Por su parte, el Ministerio de Salud expidió el "Reglamento para la regulación de los centros de recuperación para tratamiento a personas con adiciones o dependencias a sustancias psicoactivas", proponiendo un nuevo enfoque de salud pública para enfrentar el desbordado fenómeno de las drogas en el país. El uso y consumo de sustancias sujetas a fiscalización es preocupante. El plan oficial para el desarrollo nacional señala que alrededor de uno de cada cuatro adolescentes se ha embriagado, $29 \%$ de adolescentes vio a algún estudiante de su colegio usando drogas; para $15 \%$ de esta población resulta fácil conseguir marihuana y a $8 \%$ le sería fácil comprar heroína (Velasco et al., 2016). Estudios realizados mencionan que la población con mayor incidencia en el consumo de drogas proviene en $40 \%$, de hogares que pertenecen al estrato socioeconómico medio-bajo y bajo" (SENPLADES, 2017). 


\subsection{Enfoque metodológico}

Metodológicamente, se aplica el enfoque de representaciones sociales que se manifiesta en las posturas y declaraciones de los actores sociales, manifiestas en sus argumentaciones, decisiones e intervenciones, y que resulta elaborado a partir del sentido común, las ideas predominantes, conocimientos, creencias y prejuicios -paradigmas, construidos con el auxilio de la ciencia y de la técnica y circulan a través de distintos canales de comunicación (Piña \& Cuevas, 2004). Se trata de identificar rasgos definitorios en las posturas del estado y de la comunidad, para comprender mejor el significado de la serie de incendios suscitados reiterativamente. Más allá de lo circunstancial y anecdótico, los incendios (suicidios, fallecimientos) muestran un patrón de argumentos, lógicas, condicionamientos, actitudes y comportamientos, es decir de representaciones sociales que mantienen un carril propio y diferenciado.

La descripción y explicación de la problemática del consumo de drogas y, en consecuencia, la toma de decisiones e intervenciones efectivas para prevenirla-tratarla, en los diferentes ámbitos, han sido insuficientes e ineficientes, a juzgar por las tendencias de uso y abuso de las sustancias sujetas a fiscalización. Las investigaciones oficiales relevan un panorama incremental de la prevalencia secular (1998-2005) del consumo tanto en población general cuanto en población escolarizada de nivel medio y superior (Albornoz \& Puente, 2009). Los indicadores de prevalencia de vida para los consumos, disminución de la edad promedio de inicio y un nuevo menú de utilización de sustancias de mayor peligrosidad para la salud física y mental de los usuarios, evidencian la falta de impacto de las estrategias de prevención y cuestionan la efectividad de la rectoría estatal.

Este panorama negativo podría tener relación con el modelo de la prevención de la demanda de drogas, modelo fuertemente alineado con los paradigmas hegemónicos de los patrones jurídico-punitivo y médico sanitarista de la construcción social de la realidad. Este paradigma considera que, cuando se marca un cierto tipo de actividad como infamante, con el refuerzo a veces, de la sanción penal, cuando se lo estigmatiza, en la definición de Goffman, no se apunta tanto al cambio del comportamiento de los que están involucrados en dicha actividad, como a controlarlos mejor y separarlos del resto de la población, para preservar a ésta de la contaminación (Casas, 2002). Estos modelos de explicación que en la práctica no han logrado conseguir sus propósitos, se analizan como insuficientes e ineficientes en términos de su utilidad preventiva. 
Su límite está dado por su propia e inevitable propensión a procurar, como punto de partida y de llegada, la "etiologización" y "biologización" de los consumos de sustancias, asimilándolos a enfermedades infecto contagiosas. Este marco explicativo impide encontrar las causas reales del uso problemático de drogas "al negar la voluntad humana en el hecho", se utiliza como analogía, la idea del vicio en la salud mental.

El problema es que las prácticas cotidianas no siempre se ajustan a los presupuestos que impone la ley; o mejor al revés, la ley pocas veces interpreta los sentidos que tienen las prácticas. Éstas se mueven con otras lógicas, mucho más simbólicas y cargadas de sentido; por eso que la ilegalidad en que permanecen muchas de estas sustancias y la norma cultural que pesa sobre este tipo de prácticas, no alcancen como formas de control. O que ni siquiera haya resultado efectivo el recurso a la fuerza ni la imposición de penas aflictivas (Ghiardo, 2003, 125).

El corpus de conocimiento sobre el uso de drogas ha sido y es fundamentalmente positivista, modelado por los análisis tradicionales predominantemente cuantitativos. No han alcanzado a comprender el mundo que la juventud contemporánea ha configurado en el tema de las drogas en tanto (sub)cultura que asigna sentidos propios materiales y simbólicos que no siempre (o pocas veces) siguen la norma de impronta punitiva9. Desde la vertiente oficial, no se ha podido comprender de manera suficiente los modelos de representaciones sociales de los jóvenes ni de las posturas de la familia afectada sobre el tema, generándose una limitación para orientar estrategias de intervención coherentes con el momento histórico y de demanda identitaria de este grupo. En el mundo adulto, madres y padres de familia, comparten la percepción fragmentada del consumo, dentro de la corriente sanitarista y punitiva, más aún cuando el encuentro que tienen con el consumo problemático de los adolescentes y jóvenes no es temprano ni previo, sino en fases de intensa o grave adicción, ante lo cual el patrón explicativo etiológico-biologista, es parte de la representación social que guía sus decisiones. En este caso, el aislamiento del sujeto con consumo problemático de la "causa" de su adicción reducida a la sustancia como tal, y del medio social que lo indujo al "vicio", aparecen como una solución viable y como una última esperanza de curación.

\subsection{Representaciones sociales}

El enfoque de representaciones sociales posibilita un ejercicio de ordenamiento teórico sobre el tema en un escenario nacional caracterizado por: i) una información dispersa sobre

\footnotetext{
${ }^{9} \mathrm{E}$ l sentido tiene un registro que está en lo que es sentido por el sujeto como organismo (ver, palpar, etc.); pero tiene otro registro que es «subjetivo» o social, está en lo que representa para el sujeto, lo que simboliza.
} 
el uso/abuso de drogas en el país (usualmente desorganizada, insuficiente y a la vez contradictoria y discrepante); ii) la focalización del fenómeno en comunidades marginales, lo que genera juicios y opiniones no siempre apegados a la realidad, sino más bien sesgados de emocionalidad en función de la opinión interesada; y iii) la tendencia, desde la oficialidad, a un manejo de generalizaciones que le acredita para opinar, tomar posiciones, dictar normas y configurar los dispositivos de prevención, control y tratamiento de las adicciones, al intervenir sobre un fenómeno de interés público (Mora, 2002). Se propone una reflexión crítica sobre la coherencia de los modelos de reducción de la oferta y demanda de las drogas propuestos desde la oficialidad. Por otro lado, intenta una identificación y estratificación de representaciones entre grupos socioeconómicos diversos para establecer diferencias en determinadas facetas del objeto de estudio cuando estas no son tan claras (Páez, 1987 citado por Mora, 2002).

Las representaciones sociales corresponden a categorías hermenéuticas aplicadas a la gestión del uso/abuso de drogas en el contexto de la serie de incidentes, para decodificar los paradigmas, argumentos, decisiones, acciones e intervenciones relacionadas. Permiten explicar la gestión pública de lo un problema de salud. Este ejercicio podría aportar a su organización para que la población de estudio pueda orientarse en su mundo, así como posibilitar su comunicación proporcionando códigos para el intercambio social (Pérez, 2003). Se asume el enfoque conceptual de 'representación social', de Moscovici en 1979 (Moscovici, 1979):

Una modalidad particular de conocimiento, cuya función es la elaboración de los comportamientos y la comunicación entre los individuos... es un corpus organizado de conocimientos y una de las actividades psíquicas gracias a las cuales los hombres hacen inteligible la realidad física y social, se integran en un grupo o en una relación cotidiana de intercambios, liberan los poderes de su imaginación... son sistemas de valores, nociones y prácticas que proporciona a los individuos los medios para orientarse en el contexto social y material para dominarlo... (Moscovici, citado por Perera Pérez, 2003, 10)

En la concepción de Jodelet, las representaciones sociales emergen como categorías que nos ayudan a clasificar lo que ocurre en nuestro contexto, permitiéndonos así interpretar la realidad cotidiana (Jodelet, 1986). Esta visión teórica se estructura mediante un conjunto de opiniones, creencias, estereotipos, códigos, valores y normas, a las que se suman los avances en la ciencia y la tecnología. En el enfoque se localiza los objetos, personas y sucesos del contexto de los procesos de rehabilitación de los consumos problemáticos (adicciones), experimentados por estas poblaciones mediante el relevamiento de categorías 
compartidas, lo que nos permite orientar con mayor objetividad nuestra forma de pensar sobre estos colectivos (Salazar, 2003).

El sendero recorrido, lo metodológico del presente trabajo, inicia con el asombro del observador cuando se hace público el incendio de una clínica clandestina con 18 víctimas mortales. De allí, se pasa a una indagación retrospectiva de situaciones similares y se registran las reacciones de los actores sociales. Se hace un seguimiento de hemeroteca, físico y en sitios web institucionales, tales como Ministerio de Salud Pública, Gobierno Descentralizado de Guayaquil, instituciones de control de consumo problemático de sustancias, así como de periódicos de circulación nacional, local, emisoras de televisión de alcance nacional y reportes de agencias internacionales. Se recopilaron datos de medio centenar de artículos de prensa y espacios televisados. Así se hizo un primer hallazgo, el incendio referido no es único, hay una serie de casos similares que plantean un patrón que debería ser comprendido y, en lo posible, explicado.

Un segundo momento metodológico, consistió en revisar la secuencia de las respuestas de los actores sociales ante los acontecimientos en estudio. Para luego pasar a definir los constructos que motivan y se expresan en las posturas, discursos e intervenciones, es decir, dar forma a las representaciones sociales de los actores sociales. En este estudio se consideran como categorías de análisis: los constructos que explican la actitud de los actores involucrados, su orientación favorable o desfavorable con relación la rehabilitación de personas con adicciones; la organización, cantidad y calidad de la información respecto a las adicciones; y, la estructuración y organización jerárquica de los contenidos de la representación en función de factores ideológicos (Mora, 2002). Interesa aproximarse a "cómo" se producen determinadas significaciones, es decir cómo cada actor social construye una determinada visión de la realidad, privilegiando su sentido, sus categorizaciones, valoraciones y percepciones. La tarea del investigador es interpretar lo interpretado por los sujetos (Ceirano, 2000).

Se parte de la aceptación epistemológica de la no existencia de un mundo "verdadero", preexistente a los fenómenos, que operaría como el fundamento de toda explicación y desde el cual se presupondrían significaciones, así como la existencia de distintas construcciones sociales de la realidad (Berger-Luckmann 1974, Schutz-Luckmann 1977). El "mundo" es una idea del mundo y existen distintos mundos posibles en una misma realidad, como ideas de un mismo pensamiento interactuando en una pugna de poder, e implican diferentes 
representaciones e interpretaciones de lo real, guiadas por la voluntad de verdad (Ceirano, 2000) (Lahitte \& Bacigalupe, 2007).

\subsection{Representaciones paralelas}

La lectura sistemática y bajo las premisas metodológicas expuestas de la serie de incendios de clínicas de rehabilitación de personas con adicciones, entre 2016 y 2019, permite diferenciar las representaciones sociales del Estado y de la comunidad.

\subsubsection{Posicionamiento ante prevención y rehabilitación de las personas con adicciones}

- El Estado y las instituciones rectoras de la salud consideran que los únicos procesos válidos para rehabilitación son aquellos que se cumplen en los centros con licenciamiento y acreditación, reglamentada y validad por sus instancias operativas. Lo que esté por fuera, es clausurado y considerado "clandestino e ilícito". Por tanto, su funcionamiento depende de que ajuste a ciertas normas, de lo contrario merece sanción o clausura. Luego de cada uno de los incendios, la respuesta oficial fue clausurar el establecimiento que se había convertido en cenizas, clausurar los que aparecieren sin permiso en la zona del flagelo y ofrecer, mediática y reiteradamente, construir nuevos centros de rehabilitación públicos.

- La comunidad reconoce que lo necesario e importante para su familiar con adicción es el tratamiento que puede recibir en un centro de desintoxicación o clínica, independientemente de su acreditación oficial y de la "metodología de desintoxicación". Para la familia del afectado, no es condición que sea un centro acreditado pues éstos, en general, son más costosos y, en su circunstancia socioeconómica, la preferencia está marcada por su acceso económico. Así, la posibilidad (única) de rehabilitación está dada por lo que existe en su medio. Los centros son vistos como la única y última esperanza de rehabilitación de su ser querido con consumo problemático. En esta preferencia, el acceso a un servicio público sin pago de bolsillo, no tendría duda en ser la primera opción.

\subsubsection{Posibilidades de recuperación en función de la diversidad de consumos}

- Se observa una coincidencia de la representación social desde el Estado y desde la comunidad. La recuperación es un asunto individual, al que se debe atender de diversas maneras, con estrategias de internamiento, desintoxicación farmacológica, seguimiento psicoterapéutico y reinserción social. En este sentido, se trata de una enfermedad, de origen social, para la cual se han diseñado procesos terapéuticos protocolizados y de estricto cumplimiento. Esta representación social se fundamenta en el tipo de adicción y la historia individual del consumo, siendo necesario ingentes recursos que podrían generar éxito en esta estrategia programática. Por lo tanto, se recupera y rehabilita quien decide hacerlo de forma individual. La situación familiar y comunitaria, aparentemente, juega un papel secundario, se deja a un lado el peso en el proceso generador de adicciones con lo cual, todo el peso del proceso de rehabilitación recae en los hombros del individuo. 


\subsubsection{Credibilidad de los operadores de programas preventivos}

- El Estadoño reconoce la validez de procesos terapéuticos de centros de desintoxicación que no han recibido una acreditación normativa o que incumplen la norma, independientemente de su efectividad o aceptación social. Si no acatan los reglamentos, protocolos y perfiles ideados por sus técnicos-as y con base en el paradigma oficial, por ejemplo, en cuanto al tipo y número de profesionales, los centros no deben existir y son clausurados. Sin embargo, aboga por sus propios operadores o, en ocasiones, para quienes reciben su aprobación independientemente del acatamiento de sus reglas ${ }^{10}$.

- La comunidad deposita su esperanza en los métodos de recuperación que los centros de su barrio ofrecen, incluyendo prácticas represivas o lesivas a la dignidad, por ejemplo, encerrar o inclusive encadenar, a las personas que experimentan un síndrome de abstinencia. Con ocasión de alguno de los incendios, la comunidad expresó su inconformidad con la clausura de centros por parte del Estado, aunque, en otras ocasiones, los ha rechazado de manera frontal y hasta violenta ${ }^{11}$.

\subsubsection{Aceptabilidad social de proveedores de los servicios de rehabilitación}

- El Estado acepta y legaliza los servicios de rehabilitación que son licenciados y acreditados por sus normas, oficinas y operadores-as técnico-administrativos-as. Los demás son expulsados del círculo de quienes operan con validez y aceptación institucional, pasando a la lista de sujetos punibles y, sus acciones son altamente sospechosas como inadecuadas o erradas.

- La comunidad mantiene flexibilidad y apertura para aceptar proveedores que no tengan apego a la normativa técnica del Estado, la misma que, por otro lado, exige niveles de conocimiento e información que la comunidad no dispone. Frente a los servicios públicos de rehabilitación, no duda en acudir pero la demanda supera ampliamente la oferta estatal, por tanto no hay acceso. Para el Estado resulta crucial el cumplimiento de la norma, aparentemente, aunque ello significa que la mayoría de personas con adicciones no logre acceso a servicios acreditados, por tanto su única opción de acudir a centros no legalizados pero legitimados por la comunidad.

\subsubsection{Participación social en un problema de salud pública}

- En la representación social predominante, se traza el proceso de prevención y recuperación de las adicciones como un asunto ajeno a la comunidad y la familia. El Estado excluye a la comunidad de afectados y a la comunidad del escenario geodemográfico (donde se forja social y culturalmente el panorama de las adicciones), las instituciones estatales no validan ni reconocen cualquier estrategia que no cuadre con sus normas técnicas y administrativas ${ }^{12}$. La comunidad no puede participar ni es

\footnotetext{
${ }^{10}$ En octubre de 2019, se hizo público que "las tres únicas clínicas públicas para rehabilitar adictos a las drogas funcionan en Guayaquil sin licencias. Es decir que estos lugares regulados por el Ministerio de Salud Pública (MSP) están en iguales condiciones legales que las más de 73 clínicas de rehabilitación privadas que, desde enero pasado, están tratando de conseguir su licenciamiento. Pero según sus propietarios les ha resultado "imposible"... a pesar de la demanda de pacientes con adicciones, solo hay tres Centros Especializados en Consumo Problemático en Alcohol y otras Drogas (CETADS) privados que tienen la licencia. El resto está sujeto a clausuras". Fuente: Expreso, tomado de https://www.expreso.ec/guayaquil/acabo-ano-clinicas-obtuvieron-licenciamiento-2276.html

${ }^{11}$ En octubre de 2019 , luego de un incendio que provocó víctimas entre los jóvenes internados, los familiares de .alguno de los fallecidos incendió otra clínica que, decían, era propiedad del mismo dueño.

${ }^{12}$ Desde hace más de una década, aparejado al diseño y montaje de una estructura administrativa de las instituciones estatales en zonas, distritos y circuitos, que resulta paralela y sobrepuesta a la estructura política de provincias,
} 
invitada a debatir sobre el problema de salud colectiva. La comunidad, para el Estado, es beneficiaria pasiva de la intervención estatal, aunque el discurso la defina como actora participante.

- El Estado no toma en cuenta que la Atención Primaria de Salud (OMS \& UNICEF, 1978; Almeida, Artaza, Donoso, \& Fábrega, 2018; OPS/OMS, 2008), en sus versiones clásica y renovada, propone la necesidad de considerar a la comunidad como protagonista -junto a los equipos técnicos institucionales, de los procesos de salud/enfermedad, lejos de ser entendida como beneficiaria de brillantes y muchas veces costosos programas técnicos concebidos fuera de la racionalidad de su demanda.

- La comunidad afectada por adicciones, por su lado, no encuentra forma de involucrar a la población de su entorno, tanto por el estigma que implica, como porque cualquier iniciativa en este sentido no encuentra eco en el aparato estatal que, como primera condición, exige el cumplimiento de sus protocolos, normas y reglamentos.

\section{CONCLUSIONES}

El desafío metodológico en ese estudio cualitativo, ha consistido en la superación de la comprensión de la realidad en el sentido individual y unidireccional (sujeto-objeto), a un proceso colectivo en el cual las representaciones sociales sobre las adicciones, su prevención y recuperación, en dos actores sociales fundamentales: el Estado y la comunidad, son paralelas. Si bien hay elementos coincidentes que responden a paradigmas predominantes, el Estado maneja una representación social rígida y tecno-céntrica, con ceguera absoluta del sentido y necesidad de la comunidad, que no es compatible y que, en muchos casos es contradictoria con la dimensión del problema de salud pública que constituyen las adicciones.

El rumbo de esta postura estatal, argumentada desde lo técnico-administrativo, fundada en un paradigma dicotómico planteando lo individual-orgánico como eje desprendido de lo social-cultural-comunitario, es paralelo y cierra el diálogo con la representación social de la comunidad que se muestra flexible y abierta a soluciones que rebasan la respuesta estatal notablemente deficitaria, pues $89 \%$ de personas que requieren recuperación de adicciones, están avocadas a buscar su propia salida, la responsabilidad recae directamente sobre las familias y la comunidad pues, apenas $11 \%$ restante es atendido en centros públicos.

El posicionamiento estatal, sólidamente adherido a su representación social y sentido de prevención-rehabilitación dentro de su patrón y modelo, es incongruente con la dimensión humana y la carga social que la comunidad debe soportar. En consecuencia, la vida

cantones y parroquias, se observa que la tecnocracia pública sustituyó la expresión "trabajo comunitario" o "trabajo con la comunidad", son la expresión "trabajo en territorio". Esta sustitución no es gratuita, tiene una carga semántica motivo de un estudio posterior. Trabajo en territorio y trabajo en la comunidad no es lo mismo ni es igual. 
fracturada de las personas con adicciones debe esperar que algún momento haya centros legalizados suficientes, públicos o privados, pero normados. Esta espera desesperanzada no es asumida como problema de la entidad rectora. Es necesario buscar y encontrar un callejón de salida.

Se propone volver la mirada a la participación social como pilar y estrategia de la Atención Primaria de Salud. La APS, como filosofía y horizonte, como gran herramienta conceptual y práctica que permita enfrentar los problemas de salud/enfermedad, incluyendo las adicciones y la rehabilitación de las personas con consumo problemático. Se requiere considerar a la comunidad -no solo a la persona individualmente- como sujeto de la problemática y por lo mismo, como un actor social con derecho a participar de manera democrática y real -con sus recursos, ideas y aspiraciones, en la formulación e implementación de soluciones (Terán-Puente, 2019).

La participación social convoca a impulsar redes de apoyo natural y las alianzas palpitantes entre la población y los servicios existentes, aún los denominados "clandestinos" desde la formalidad estatal, entendiendo el carácter de la asociación no escrita, haciéndola parte de una solución con procesos de contexto y superación de las limitaciones reales de la institucionalidad y de la "ilegalidad". Solo así, seguramente, se podrá hacer frente al crecimiento exponencial de las adicciones y a la proliferación de servicios sin control de calidad que debe ser medida desde y con la comunidad y no, excluyentemente, por un aparato estatal a todas luces rebasado en su capacidad y calidad de respuesta, por tanto, absolutamente cuestionable en su capacidad rectora.

Sin participación social plural, efectiva, viva y realista -alrededor de los problemas palpitantes, vivos, reales- no es posible la rectoría del estado y la APS se mantendrá en el limbo de modelos inalcanzables, aunque se la renueve, reitere y reafirme en los foros internacionales.

Es vital la eliminación de las barreras entre el aparato estatal y la comunidad, dentro de un paradigma nuevo, que ponga la verdad en el centro de la comunidad y no en el escritorio de la autoridad. Así, una rectoría de otro tipo será posible. Esto significa un golpe de timón al paradigma biomédico y a la caduca democracia representativa que excluye, criminaliza y declara clandestino lo que no puede controlar bajo su norma técnico-administrativa especializada en una intervención punitiva y controladora que pone el foco en sí misma y no en los problemas de salud pública. 
La ruptura de paradigmas significaría ampliar las representaciones sociales desde el estado con variables hasta hora no aceptadas, como la única forma para lograr el control efectivo de la expansión del universo que lleva al consumo problemático, superando posturas que no han funcionado para la comunidad -el micro tráfico y el macro tráfico son inversiones boyantes de lucro ilimitado para los carteles y para los sistemas financieros.

No es posible concluir este estudio sin señalar que el costo de la rectoría fallida es, lamentablemente, la pérdida de vidas humanas, víctimas sin derecho a una rehabilitación por inexistencia de servicios seguros y accesibles. Mientras se mantenga el modelo representativo y excluyente de democracia -amparada por una aparataje estatal impresionante separado de la comunidad- seguirán habiendo víctimas de la expansión de las adicciones, seguirán los vacíos en prevención y rehabilitación de las personas con adicciones y se mantendrán las brechas en los derechos de las personas.

En América Latina, a pesar del predominio aplastante de la sociedad de mercado y de sus expresiones en el Estado, se han gestado procesos comunitarios, realmente participativos, que enfrentan los problemas sociales con la comunidad, mucho más allá de lo normativoadministrativo. Una de las expresiones de esta búsqueda es el movimiento de terapia comunitaria sistémica, cuya metodología es simple y rigurosa, preserva la actoría comunitaria, abre el entendimiento y el compromiso a soluciones compartidas que, desde la tecnocracia permanecen invisibles, imposibles e inaceptables ${ }^{13}$.

Es hora de indagar propuestas de nuevo tipo y de largo alcance. E. Granda, junto con Morin $(1994,1997)$, resalta la evidencia de que la sociedad alcance momentos de "ruptura integradora" para cambiar el rumbo societario, ruptura que la define como "momento en el cual dice ¡basta!, e inicia un proceso de exorcismo de los demonios del ayer, afilia las voluntades de todos sus hijos, descubre y desarrolla sus potencialidades internas, neutraliza los obstáculos externos y decide parecerse a sí misma. La historia de los pueblos tiene momentos de reflexión-acción importantes que los llevan a reencontrarse..." (Granda, 2009). La conservación de las representaciones sociales paralelas entre el Estado y la comunidad, evidenciadas en este estudio, mantendrán los problemas de salud pública en un callejón, sin salida. La salud colectiva, como proceso social, seguirá siendo un pendiente.

\footnotetext{
${ }^{13}$ La Terapia Comunitaria Integrativa, ideada por Adalberto de Paula Barreto, tiene más de dos décadas de construcción metodológica y de acción comunitaria, ligada a la comprensión de Paulo Freire en educación popular, al constructivismo y a un sentido humanista fundacional, propone una metodología que facilita y promueve compartir las representaciones sociales para desarrollar nuevos saberes, soluciones y compromisos desde el corazón y en la vivencia de la comunidad (Barreto, A. 2015)
} 


\section{REFERENCIAS}

Almeida, G., Artaza, O., Donoso, N., \& Fábrega, R. (2018). La atención primaria de salud en la Región de las Américas a 40 años de la Declaración de Alma-Ata. Revista Panamericana de Salud Pública. https://doi.org/10.26633/RPSP.2018.104

Asamblea Nacional del Ecuador. (2004). Código Orgánico Integral Penal. (D. Hu. y C. Ministerio dde Justicia, Ed.), Ministerio de Justicia, Derechos HUmanos y Cultos (Vol. 30). Quito. https://doi.org/10.1111/j.15591816.2000.tb02505.x

Casas, A. A. (2002). Drogadicción, salud y política. Herramientas. Retrieved from https://herramienta.com.ar/autores.php

Ceirano, V. (2000). Las representaciones sociales de la Pobreza. Cinta de Moebio, 9, 1-18.

Ecuador, A. C. (2008). Constitución de la República del Ecuador.

Filho, N. A., \& Paim, J. S. (1999). La crisis de la salud pública y el movimiento de la salud colectiva en Latinoamérica. Cuadernos Médico Sociales, 75, 5-30.

Ghiardo, F. (2003). Acercandonos al sentido del uso de drogas y la prevencion desde los jovenes. Ultima Década, 18, 123-151.

Granda, E. (2004). ¿A qué llamamos salud colectiva, hoy? Revista Cubana de Salud Pública, 30(2), 14.

Granda, E. (2009). Compromiso Social de la Investigación en Salud. La Salud y La Vida 2, 2, 29-39. Retrieved from http://www.alames.org/index.php/documentos/libros/medicina-social/libros-1/68-la-salud-y-la-vidavolumen-2/file

Gross Albornoz, M., \& Terán Puente, J. M. (2009). Representaciones sociales de los jóvenes ecuatorianos sobre el uso de drogas. Quito.

Jodelet, D. (1986). La representación social : fen ómenos, concepto y teoría.

Lahitte, H., \& Bacigalupe, M. de los Á. (2007). Observación y conocimiento: Relevancia del investigador en la construcción de la Antropología como ciencia cognitiva. Fermentum. Revista Venezolana de Sociología y Antroólogía, 17(49), 13.

Mora, M. (2002). La teoria de las representaciones sociales de Serge Moscovici. Athenea Digital, $2(4), 782$. https://doi.org/10.2307/2802632

Moscovici, S. (1979). LA REPRESENTACIÓN SOCIAL: UN CONCEPTO PERDIDO. Ed. Huemul, Buenos Aires, 27-44. Retrieved from https://s3.amazonaws.com/academia.edu.documents/32383883/Mosvici_cap_1_Psicoanalisis.pdf?AWSAcc essKeyld=AKIAIWOWYYGZ2Y53UL3A\&Expires=1522635504\&Signature=5hkV7Zjx7EoTAEJ3QbTAp6a2L ow\%3D\&response-content-disposition=inline\%3B filename\%3DIEP_-Instituto_de_Es

MSP. (2019). Centros especializados en tratamiento a personas con consumo problemático. Quito.

Ops;Oms. (2008). La Renovación de la Atención Primaria de Salud en las Americas. La Renovación de La Atención Primaria de Salud En Las Americas, 1-46. https://doi.org/ISBN 978-92-75-33262-7 -

Perera Pérez, M. (2003). A PROPÓSITO DE LAS REPRESENTACIONES SOCIALES. APUNTES TEÓRICOS, TRAYECTORIA Y ACTUALIDAD. La Havana: CLACSO.

Piña, J., \& Cuevas, J. (2004). La teoría de las representaciones sociales. Su uso en la investigación educativa en México. Perfiles Educativos, XXVIII, 12.

Rodríguez Salazar, T. (2003). El debate de las representaciones sociales en la psicología social. Relaciones, Estudios de Historia y Sociedad, XXIV(93), 17.

Secretaría Técnica de Drogas. (2017). Costo del fenómeno de las drogas en Ecuador en 2015 Subsecretaría Técnica de Análisis, Prospectiva y Evaluación Elaborado por: MSc. Fernando Naranjo y MSc. David Jaramillo Quito , Ecuador Contenido. Quito. Retrieved from http://www.cicad.oas.org/oid/pubs/EconomicCostofDrugsEcuador2015ESP.pdf

SENPLADES. (2017). Plan Nacional de Desarrollo 2017-2021. (E. S. Secretaría Nacional de Desarrollo, Ed.) (SENPLADES). Quito: SENPLADES.

Terán-Puente, C. (2019, January). 18 VIDAS INCINERADAS EN CLINICA “CLADESTINA.” La Lñinea de Fuego. Retrieved from lalineadefuego.info 